\title{
Oxidative stress, cell cycle, and neurodegeneration
}

\author{
Jeffrey A. Klein and Susan L. Ackerman \\ The Jackson Laboratory, Bar Harbor, Maine, USA \\ J. Clin. Invest. 111:785-793 (2003). doi:10.1172/JCI200318182.
}

While numerous studies have examined the existence of increased reactive oxygen species (ROS) in later-onset neurodegenerative disorders, the mechanism by which neurons die under conditions of oxidative stress remains largely unknown. Fairly recent evidence has suggested that one mechanism linked to the death of terminally differentiated neurons is aberrant reentry into the cell cycle. This phenomenon has been reported in Alzheimer disease (AD) patients (1), Down syndrome patients (2), and several mouse neurodegenerative models (3-5). We will discuss recent findings regarding the influence of oxidative stress on neurodegeneration and possible connections between oxidative stress and unscheduled cell cycle reentry, the understanding of which could lead to new strategies in the development of therapeutic agents for neurodegenerative disorders.

\section{Oxidative stress and neuron death}

Under normal physiological conditions, it is estimated that up to $1 \%$ of the mitochondrial electron flow leads to the formation of superoxide $\left(\mathrm{O}_{2}{ }^{-}\right)$, the primary oxygen free radical produced by mitochondria; and interference with electron transport can dramatically increase $\mathrm{O}_{2}{ }^{-}$production. While these partially reduced oxygen species can attack iron sulfur centers in a variety of enzymes, $\mathrm{O}_{2}{ }^{-}$is rapidly converted within the cell to hydrogen peroxide $\left(\mathrm{H}_{2} \mathrm{O}_{2}\right)$ by the superoxide dismutases (SOD1, SOD2, and SOD3). However, $\mathrm{H}_{2} \mathrm{O}_{2}$ can react with reduced transition metals, via the Fenton reaction, to produce the highly reactive hydroxyl radical $\left({ }^{\circ} \mathrm{OH}\right)$, a far more damaging molecule to the cell. In addition to forming $\mathrm{H}_{2} \mathrm{O}_{2}, \mathrm{O}_{2} \cdot-$ radicals can rapidly react with nitric oxide $(\mathrm{NO})$ to generate cytotoxic peroxynitrite anions $\left(\mathrm{ONOO}^{-}\right)$. Peroxynitrite can react with carbon dioxide, leading to protein damage via the formation of nitrotyrosine and lipid oxidation.

\footnotetext{
Address correspondence to: Susan L. Ackerman, The Jackson Laboratory, 600 Main Street, Bar Harbor, Maine 04609, USA. Phone: (207) 288-6494; Fax: (207) 288-6077; E-mail: sla@jax.org. Conflict of interest: The authors have declared that no conflict of interest exists.

Nonstandard abbreviations used: reactive oxygen species (ROS); Alzheimer disease (AD); superoxide $\left(\mathrm{O}_{2}{ }^{--}\right)$; hydrogen peroxide $\left(\mathrm{H}_{2} \mathrm{O}_{2}\right)$; superoxide dismutase (SOD); $\beta$-amyloid (A $\left.\beta\right)$; substantia nigra (SNc); Parkinson disease (PD); cyclin-dependent kinase $(\mathrm{CDK})$; proliferating cell nuclear antigen (PCNA); 8-hydroxydeoxyguanosine (8-OHdG); extracellular signal-regulated kinase (ERK).
}

The generation of ROS in normal cells, including neurons, is under tight homeostatic control. To help detoxify ROS, biological antioxidants, including glutathione, $\alpha$-tocopherol (vitamin E), carotenoids, and ascorbic acid, will react with most oxidants. In addition, the antioxidant enzymes catalase and glutathione peroxidase detoxify $\mathrm{H}_{2} \mathrm{O}_{2}$ by converting it to $\mathrm{O}_{2}$ and $\mathrm{H}_{2} \mathrm{O}$. However, when ROS levels exceed the antioxidant capacity of a cell, a deleterious condition known as oxidative stress occurs. Unchecked, excessive ROS can lead to the destruction of cellular components including lipids, protein, and DNA, and ultimately cell death via apoptosis or necrosis (6).

Although numerous in vitro studies have implicated ROS in neuronal death (7), the relative lack of in vivo evidence has contributed to some controversy surrounding the role of ROS in the pathophysiology of later-onset neurodegenerative disorders. Mice homozygous for a targeted mutation of the $\alpha$-tocopherol transfer protein gene $(\alpha-T t p)$ develop retinal degeneration and gait abnormalities at 1 year of age, correlated with degeneration of the posterior column of the spinal cord (8). These behavioral and pathological changes were largely prevented with $\alpha$-tocopherol supplementation. In addition, $\mathrm{Sod}^{-/-}$mice develop gait abnormalities at 12 days of age, associated with vacuolization in brainstem and cortical regions (9). These results indicate that the loss of antioxidant genes can lead to neuron loss.

Markers of oxidative stress are found in postmortem examination of brains from patients with many neurodegenerative disorders (10). However, whether oxidative stress is involved in the development and/or progression of these disorders, or is merely associated with end-stage disease, is in dispute. DNA oxidation, protein oxidation, and lipid peroxidation have been reported in regions containing neurofibrillary tangles and senile plaques of brains from $\mathrm{AD}$ patients (10). As an apparent compensatory response, increased levels of catalase, glutathione peroxidase, and glutathione reductase were observed by RT-PCR from the hippocampus and inferior parietal lobe of brains of patients with $\mathrm{AD}$ (11). Biochemical assays for salicylate hydroxylation using $\beta$-amyloid $(A \beta)$ fragments, the primary constituent of senile plaques, suggest that these peptides can generate free radicals that may underlie some of the molecular alterations observed in $\mathrm{AD}$ brains $(12,13)$. In agreement, increases in $A \beta$ deposition resulted in the induction of oxidative stress in transgenic mice overexpressing the 
mutant amyloid precursor protein and presenilin 1, two proteins implicated in the progression of $\mathrm{AD}(14)$. These results suggest that $A \beta$ may be involved in free radical generation. However, other studies suggest that $A \beta$ may be a cellular response to oxidative stress or an antioxidant and implicate other processes as primary in generating free radical damage (15).

Dopaminergic neurons in the substantia nigra $(\mathrm{SNc})$ of brains of Parkinson disease (PD) patients also exhibit hallmarks of oxidative stress, including lipid peroxidation, nucleic acid and protein oxidation, and changes in some antioxidants (16). Furthermore, $\alpha$-synuclein, the main deposition product in inclusions in the SNc of PD patients, is a specific target of nitration, suggestive of the role of oxidative damage in the formation of these inclusions (17). In agreement, in vitro studies have shown that these aggregates are stabilized by oxidation. These findings, and the intrinsic potential for the oxidative metabolism of dopamine to generate ROS, have suggested that oxidative stress may be involved in the death of neurons in the SNc of these patients. In addition, 1-methyl-4phenyl-1,2,3,6-tetrahydropyridine, rotenone, and paraquat, compounds that can generate ROS and/or disrupt the electron transport chain, have been found to induce symptoms of PD in animal models, including, in some cases, the deposition of $\alpha$-synuclein aggregates (18-20). These substances also represent environmental risk factors for PD (21).

Oxidative damage has also been reported in several other age-related neurodegenerative diseases, including Huntington disease, progressive supranuclear palsy, amyotrophic lateral sclerosis, and prion disorders, all of which have abnormal protein aggregation as a major component of their pathology (22-24). Although recent research had questioned the relationship between protein fibril formation and disease, it is also clear that some sort of protein aggregation plays an important role in the pathologies of these various diseases and that oxidative stress and protein inclusions may cooperate in neuronal death in several neurodegenerative disorders.

Intrinsic oxidative stress, and presumably cell damage, increase with age due to either diminished antioxidant defenses or the increase in mitochondrial dysfunction (25). These age-related effects, compounded with genetic and environmental risk factors, and the high energy dependence but relatively low level of antioxidants in the brain, may provide a unifying mechanism for the high incidence of neurodegenerative disorders in the aged population.

\section{Cell cycle and neuron death}

The misregulation of the cell cycle in many cell types can lead to unchecked proliferation and neoplastic disease. As in other cell types, the cell cycle in the CNS is tightly regulated. Neuronal precursors proceed through the cell cycle to produce larger numbers of neurons than are needed, and excess neurons are eliminated by selective apoptosis. However, once neurons are terminally differentiated, they are maintained in a quiescent G0 state and no longer cycle.
A decade ago it was hypothesized that cell cycle abnormalities may be intimately connected to the death of terminally differentiated neurons $(26,27)$. The bases for this hypothesis included the observation that tumors arising from terminally differentiated neurons are extremely rare, indicating that these cells are resistant to neoplastic transformation. Experimental evidence for this hypothesis included the demonstration that forced expression of oncogenes in terminally differentiated cells, including neurons, can cause cell death rather than cell proliferation (27).

In vitro studies have examined the link between aberrant cell cycle reentry and neuronal cell death in an attempt to better understand the mechanisms by which neurons can be forced back into the cell cycle. Postmitotic chick retinal neurons cultured in the presence of nerve growth factor, insulin, and neurotrophin- 3 underwent apoptosis via cell cycle reentry, characterized by an increase in cyclin B2 expression (28). Treatment of these cells with roscovitine, a cyclin-dependent kinase (CDK) inhibitor, prevented cell cycle reentry and apoptosis. These results demonstrate that although at least some postmitotic neurons retain the capacity to respond to growth factors by reentering into the cell cycle, such stimulation causes apoptosis rather than proliferation.

Unscheduled cell cycle reentry can also be induced by neurotoxic insults. For example, kainic acid-treated cerebellar granule cells upregulate the cell cycle proteins CDK2, cyclin E, and E2F-1 and replicate their DNA prior to apoptosis (29). Similarly, aberrant cell cycle reentry and subsequent apoptosis were observed when rat embryonic cortical neurons were cultured with toxic concentrations of $A \beta$ peptides (30). These neurons showed increased expression of cyclin D1, cyclin E, and cyclin A, as well as increased phosphorylation of the retinoblastoma $(\mathrm{Rb})$ protein. In addition, $\mathrm{A} \beta$ treatment increased the amount of the ganglioside GD3, a component of the sphingolipid signaling system known to affect neuronal proliferation and differentiation, and reduction of GD3 prevented cell cycle reentry and apoptosis (31).

Several animal models directly or indirectly support the hypothesis that aberrant cell cycle reentry leads to neuronal death. Expression of SV40 T antigen in cerebellar Purkinje cells or retinal photoreceptors of transgenic mice causes unscheduled cell cycle reentry and cell death $(27,32,33)$. Dying cells expressed high levels of proliferating cell nuclear antigen (PCNA) and had undergone DNA synthesis. Activation of G1- and Sphase proteins occurs prior to cell death in dorsal root ganglion cells from mice lacking neurotrophin-3 (34). Apoptosis could be almost entirely eliminated by injections of olomucine, a G1-phase inhibitor. In the ischemia/reperfusion stroke model in both mice and rats, cerebral neurons were observed to reenter the cell cycle prior to death $(35,36)$. Cyclin D1 activation and a loss of cyclin-dependent kinase inhibitors accompanied the cell death. The treatment of primary rat neuron cultures from stroke-induced animals with cyclindependent kinase inhibitors significantly reduced in vitro cell death. In the spontaneous early-onset neurodegenerative mouse mutants staggerer (Rorasg), 
Lurcher $\left(G r i d 2^{L c}\right)$, and weaver $\left(K c n j 6^{w v}\right)$, cell cycle reentry has been observed to occur in postmigratory granule cells prior to apoptosis $(3,4)$. This has been correlated with the expression of cell cycle proteins PCNA, CDK4, cyclin D, and cyclin A, as well as with incorporation of the DNA analog BrdU. These models support the idea that cell cycle reentry underlies neuronal apoptosis in vivo as well as in vitro.

Recent evidence suggests that cell cycle reentry precedes neuronal apoptosis in human neurodegenerative diseases as well. Recently, increases in the cell cycle regulators cyclin D1, CDK4, hyperphosphorylated pRb, and E2F-1 were observed in both spinal motor neuron and the motor cortex of postmortem tissue of ALS patients (37). Neuronal loss in the cerebral cortex of Down syndrome patients has also been correlated with overexpression of both mitogenic and differentiation signals (2). Hippocampal pyramidal neurons from the brains of these patients exhibited increased levels of CDK4, an important regulator of the cell cycle. These same neurons with increased CDK4 expression also contained neurofibrillary tangles and granulovacuolar degeneration, suggesting a correlation between cell cycle reentry and pathology.

An increase in cell cycle proteins, including PCNA, cyclin D, CDK4, and cyclin B1, has been observed in the hippocampus, as well as other diseased brain regions, from postmortem tissue of $\mathrm{AD}$ patients $(38,39,40)$. These proteins were not observed in nondiseased brain regions or in control-patient brains. In addition, FISH analysis of the hippocampus and basal forebrain of $\mathrm{AD}$ patient brains revealed many tetraploid neurons, indicating that these neurons had undergone approximately a full round of DNA synthesis (41). Furthermore, the cell cycle-associated protein Ki-67, detected in numerous types of tumors, has been observed in $\mathrm{AD}$ patient brains, as well as in patients with Pick disease and intractable temporal lobe epilepsy (42).

Data from these studies and others support the idea that mitogenic signals may lead to an increase in cell cycle proteins, thus driving neurons into aberrant cell cycle reentry. Furthermore, it is clear that when terminally differentiated neurons are forced into the cell cycle, they do not proliferate - they die. Although the mechanisms by which cell cycle reentry causes cell death are unknown, a direct link between cell cycle and neuronal death was recently made with the observation that $\mathrm{CDC} 2$, a cell cycle regulator, induces the phosphorylation and activation of BAD, a trigger of apoptosis (43).

Connections between oxidative stress and cell cycle The multiple demonstrations of biomarkers of oxidative stress in many age-related neurodegenerative disorders, combined with the more recent reports of cell cycle abnormalities in neurons from these patients, suggest that these processes may be intertwined at the molecular level. At first, the idea of oxidative stress and cell cycle reentry seems counterintuitive. In fact, examination of the current literature on the effect of oxidative stress on the cell cycle reveals that increases in ROSinduced DNA damage are correlated with cell cycle arrest (44). However, whether ROS-exposed cells under- go growth arrest or apoptosis may depend in part on where the cell resides in the cell cycle when insulted. For example, human fibroblasts treated with $\mathrm{H}_{2} \mathrm{O}_{2}$ underwent either cell cycle arrest or apoptosis. The majority of the apoptotic fibroblasts were in the S phase of the cell cycle, whereas growth-arrested cells were predominantly in the $\mathrm{G} 1$ or the $\mathrm{G} 2 / \mathrm{M}$ phase (45).

This apoptotic death of fibroblasts in the $S$ phase is consistent with the death of neurons that have aberrantly reentered the cell cycle. Dorsal root ganglion neurons from neurotrophin-3 mutant mice override the G1 phase cell cycle restriction point but die by apoptosis in the $S$ phase (34). In addition, hippocampal pyramidal and basal forebrain neurons from $\mathrm{AD}$ brains exhibit chromosomal duplication but do not undergo mitosis prior to cell death, consistent with cell death in the $S$ or G2 phase of the cell cycle (41). These results suggest that neurons are influenced by the same cell cycle checkpoints that govern apoptosis in other cell types.

Recently, we described a novel genetic mouse model that may begin to unravel the mechanisms by which oxidative stress stimulates quiescent neurons to leave the G0 phase and reenter the cell cycle prior to S-phase removal. The X-linked harlequin $\left(P d c d 8^{H q} ; \mathrm{Hq}\right)$ mutation was first identified by the almost complete absence of hair in hemizygous males and homozygous females carrying this spontaneous mutation (46). In addition to alopecia, these mice develop progressive ataxia beginning at $4-5$ months of age $(5,47)$. The onset of ataxia is correlated with apoptosis of cerebellar granule cells, the major interneurons in the cerebellum. In addition to cerebellar neuron loss, aged $\mathrm{Hq}$ mutant mice have progressive retinal degeneration, as demonstrated clinically by electroretinography (ERG) and also by histological examination. Cells in the ganglion cell layer (both retinal ganglion cells and displaced amacrine cells) are lost beginning at 3 months of age. The loss of ganglion cells leads to hypoplasia of the optic nerve. Subsequently, there is loss of both horizontal and amacrine cells in the inner nuclear layer, and of photoreceptors in the outer nuclear layer. Clinically, both rod and cone ERG responses are greatly attenuated in mutant mice by 4 months of age.

A positional cloning strategy identified the $\mathrm{Hq}$ mutation as an ecotropic leukemia proviral insertion into the first intron of the apoptosis-inducing factor gene, Aif, also known as programmed cell death 8 (Pdcd8). This insertion causes aberrant splicing from exon 1 into the provirus and a corresponding decrease of $80-90 \%$ in Aif mRNA and protein. AIF is a ubiquitously expressed $57-\mathrm{kDa}$ flavoprotein, containing a flavin adenine dinucleotide prosthetic group (48). The large C-terminal domain of AIF is similar to several plant and bacterial oxidoreductases, particularly semidehydroascorbate and ascorbate reductases and NADHdependent ferredoxin reductases. Recent determination of the crystal structure of AIF demonstrates a glutathione reductase-like fold and high similarity to biphenyl dioxygenase (49).

Under normal physiological conditions, AIF is located in the intermembrane space of the mitochondria. However, during apoptosis, AIF translocates from the 


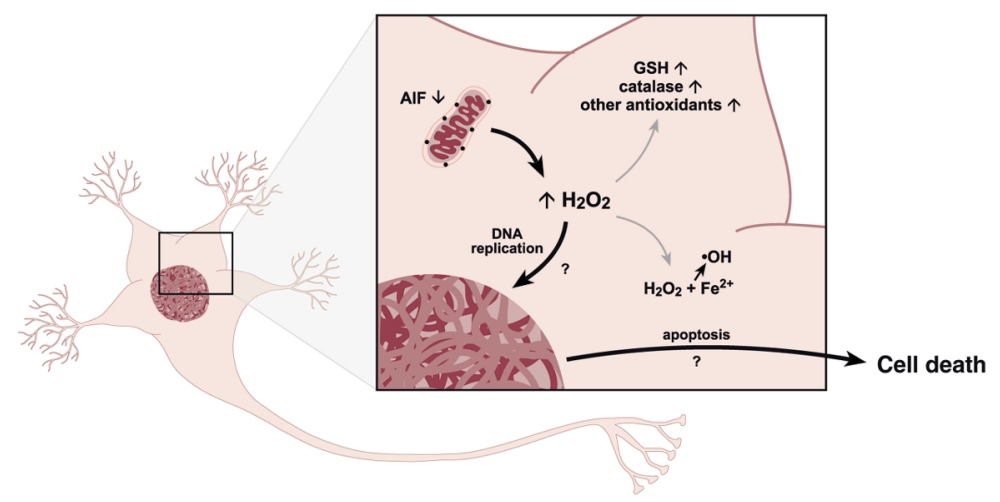

Figure 1

Oxidative stress and cell cycle reentry lead to cell death in the harlequin mouse. An $80 \%$ reduction of AIF protein expression in the $\mathrm{Hq}$ mutant mouse is associated with increased activity of total glutathione $(\mathrm{GSH})$ and catalase, presumably through increases in hydrogen peroxide $\left(\mathrm{H}_{2} \mathrm{O}_{2}\right)$. Surviving neurons may express additional antioxidant pathways. Increases in $\mathrm{H}_{2} \mathrm{O}_{2}$ could lead to cellular damage via formation of hydroxyl radicals ( $\left.{ }^{\circ} \mathrm{OH}\right)$ by the Fenton reaction. In addition, oxidative stress may trigger cell cycle reentry in some terminally differentiated neurons. These neurons undergo apoptosis after DNA replication. Possible mechanisms for cell cycle reentry are shown in Figure 2.

mitochondria to the cytoplasm and nucleus (48). This redistribution of AIF can be induced by several apoptotic paradigms in many different cell types, including primary neurons (50). Furthermore, cultured cells undergo apoptosis when transfected with AIF lacking the N-terminal mitochondrial targeting sequence or when recombinant AIF is injected into the nucleus or cytoplasm (48). The apoptogenic activity of AIF is independent of its redox activity and is not suppressed by caspase inhibitors in some mammalian cells (51-53), although genetic evidence in Caenorbabditis elegans, isolated mammalian mitochondria, and transiently transfected cells suggests that AIF release is caspase-dependent.

In further support of a proapoptotic role for AIF, exposed targeted ES cells hemizygous for a null mutation in the Aif gene were resistant to serum starvation but not to other apoptosis inducers (54). Examination of embryoid bodies produced by the in vitro aggregation of these cells demonstrated that these structures failed to undergo cavitation, suggesting a proapoptotic role for AIF in early development. However, chimeric mice could not be produced from Aif $^{-/ Y}$ ES cells, so the normal physiological function of mitochondrially localized AIF remains unknown.

As mentioned above, AIF has strong structural homology to glutathione reductase, well established as an essential component of the $\mathrm{H}_{2} \mathrm{O}_{2}$-scavenging glutathione system. This, combined with previous observations of upregulation of oxidative stress markers in many types of adult-onset neurodegeneration, suggested that the reduction in AIF in $\mathrm{Hq}$ animals may cause oxidative stress, via direct or, more likely, indirect regulation of $\mathrm{H}_{2} \mathrm{O}_{2}$. Consistent with an increase in intracellular $\mathrm{H}_{2} \mathrm{O}_{2}$, we observed increased activity of the hydrogen peroxide scavenger catalase, and total glutathione, an essential electron donor for the reduction of hydroperoxides, in cerebellar extracts from $\mathrm{Hq}$ mutants at 4 weeks of age, months before the onset of neurodegeneration (Figure 1). However, increases in levels of SOD1 and SOD2, superoxide scavengers, were not detected in mice at either age. Other hallmarks of oxidative stress were also observed in $\mathrm{Hq}$ mutant mice. Increased lipid peroxidation was found in the cerebellum and the rest of the brain in $\mathrm{Hq}$ mutant mice at 1 month of age. Furthermore, DNA oxidation, as evidenced by the presence of the modified base 8-hydroxydeoxyguanosine (8-OHdG), was noted in many neuron types, including cerebellar granule cells and retinal ganglion, amacrine, and horizontal cells.

In vitro studies of cerebellar granule cell cultures from $\mathrm{Hq}$ mutant mice demonstrated that while these cells were less sensitive to serum starvation-induced apoptosis, as predicted by studies on AIF-null ES cells, they were more sensitive to $\mathrm{H}_{2} \mathrm{O}_{2}$. This sensitivity could be rescued by retroviral transfection with Aif cDNA containing the $\mathrm{N}$-terminal mitochondrial localization signal. Interestingly, wild-type granule cells transfected with the same retrovirus were more resistant to peroxide-induced cell death, suggesting that increased amounts of AIF may actually be protective against oxidative stress-induced cell death in some instances.

The mechanism by which AIF can act to reduce oxidative stress in vivo is still puzzling at the biochemical level. In vitro, AIF has NADPH oxidase activity that, like other NADH oxidases, generates superoxide radicals (51). The loss of this activity would not be expected to generate an increase in free radicals but could in fact reduce free radical production. However, whether NADPH is the in vivo substrate of AIF is unknown (55). Although not yet tested, it has been hypothesized that AIF may play a role in the mitochondrial respiratory chain (56). If so, a dramatic reduction of AIF could increase free radical production via defects in mitochondrial respiration. Alternatively, AIF may participate in an unknown redox cycling or coupling pathway (56). Such a role would be consistent with the antioxidant activity of glutathione reductase, a structural relative of AIF, which can mediate $\mathrm{H}_{2} \mathrm{O}_{2}$ activity both through the recycling of glutathione or by coupling with the antioxidant functions of the glutaredoxin system (57). 
Because of the association of oxidative stress and abnormal cell cycle checkpoint function and reports of aberrant neuronal cell cycle reentry in progressive human neurodegenerative diseases, we investigated whether neurons in the $\mathrm{Hq}$ mutant cerebellum and retina had reentered into the cell cycle. Indeed, in older $\mathrm{Hq}$ animals many cerebellar granule cells and retinal ganglion, amacrine, and horizontal cells had newly synthesized nuclear DNA, as demonstrated by BrdU incorporation (Figure 1). These cells also expressed PCNA and CDC47, both markers of $\mathrm{S}$ phase. Consistent with studies of postmortem tissue from patients with neurodegenerative disorders, no evidence of mitotic figures in any of these cell types was observed. The nuclei of many of these cells were clearly pyknotic, and most of the cells were also positive for activated caspase- 3 , indicating that cell cycle reentry in these neurons was associated with apoptosis. Furthermore, all cycling neurons were positive for $8-\mathrm{OHdG}$, demonstrating oxidative stress-induced DNA damage. However, not all 8-OHdG-positive cells were in $\mathrm{S}$ phase, a result that may indicate a temporal relationship between oxidative stress and cell cycle. These observations and the detection of oxidative stress markers long before neurodegeneration demonstrate that induction of unscheduled cell cycle reentry is highly correlated with, and may be induced by, oxidative stress.

While many dying neurons in the $\mathrm{Hq}$ mutant mouse abnormally reenter into the cell cycle, other neurons that degenerate do not. For example, both Purkinje cells in the cerebellum and retinal photoreceptors are progressively lost but do not stain for cell cycle markers. Interestingly, these neurons are not positive for 8-OHdG nor activated caspase-3, and electron microscopy suggests that Purkinje cells, at least, undergo necrosis rather than apoptosis. Furthermore, the loss of AIF expression in many other CNS neurons (including cortical neurons) does not lead to death, even in $\mathrm{Hq}$ mutant mice aged to 2 years.

These studies clearly indicate that various types of neurons respond differently to the downregulation of AIF (and presumably the increase in free radicals). Some neurons undergo apoptosis correlated with cell cycle reentry, whereas others die without signs of either process. Furthermore, those neurons that survive may be more resistant to oxidative stress. We (5) and others (58) have observed that sensitivity to oxidative stress differs between neuron types. Cerebellar granule cells exposed to a 15 -minute pulse of $100 \mu \mathrm{M} \mathrm{H}_{2} \mathrm{O}_{2}$ displayed only a $25 \%$ survival rate after 15 hours in culture. In contrast, a similar number of cortical neurons survived when exposed to $100 \mu \mathrm{M} \mathrm{H}_{2} \mathrm{O}_{2}$ for 24 hours (58), suggesting that granule cells are more sensitive to oxidative stress than are cortical neurons. Therefore, $\mathrm{Hq}$ mutant mice provide a model to examine both the influence of oxidative stress on cell cycle reentry and the mechanisms underlying the differential response of different types of neurons to oxidative stress.

\section{Mechanisms of oxidative stress-induced cell cycle reentry}

Although both oxidative stress and cell cycle reentry have been implicated in the onset of later-onset neu- rodegenerative diseases and clearly occur together at the cellular level in $\mathrm{Hq}$ mutant mice, the mechanism by which oxidative stress may lead to cell cycle abnormalities remains unknown.

Cumulative DNA damage caused by endogenous free radicals has been suggested to underlie cancer and other age-related disorders, including neurodegeneration $(44,59)$. Can this accumulation of mutations over time play a major role in cell cycle-induced neuronal apoptosis? The progressive accumulation of oxidativedamaged DNA and the temporal increase in cell cycle reentry in the retina and cerebellum of aging $\mathrm{Hq}$ mutant mice are consistent with such a theory. The amount of 8-OHdG, a major DNA lesion resulting from free radical attack that has been shown to alter the base-pairing properties of guanine in in vitro assays, is elevated in nuclear and mitochondrial DNA in neurons in diseased brain regions of patients with neurodegenerative disorders. Furthermore, studies have shown that increases in this modified base are correlated with increased incidence of cancer (and therefore cell cycle abnormalities) (60). In addition to base modifications, oxidative stress can cause other potentially mutational events including strand breaks, discontinuous loss of heterozygosity, and large deletions (61). Thus, if the oxidation of DNA surpasses the DNA-repair capacity of the cell, mutations could accumulate, leading to the loss of genome stability. Like malignant transformation, cell cycle reentry in neurons could require somatic mutation of a complex group of genes. Furthermore, like cancer, the genetic alterations required to cause cell cycle reentry may be specific to particular cell types.

On the other hand, there is little direct evidence for mutation accumulations in aging neurons. Most direct measurements of mutation frequencies have quantified rates in replicating cell types using specific locus tests, such as the mutation rate of the selectable hypoxanthine phosphoribosyl transferase gene, Hprt (59). Recently, however, a method for the measurement of mutation frequency during aging in postmitotic tissues has been performed in transgenic mice bearing copies of lac $Z$ reporter plasmids at defined positions in the genome (62). As measured by this system, the frequency of spontaneous mutations was increased in liver between 4 and 23 months of age, and large deletions of adjacent mouse genomic material exponentially increased in animals over 23 months of age. However, such age-related increases in mutation frequency were not seen in plasmids rescued from the brains of these mice. This study did not find evidence for an increased mutation rate in the aging mouse brain; however, only alterations at, or adjacent to, the integrated transgenes were surveyed. Furthermore, large deletions in neurons may be more detrimental to the survival of neurons, and thus, cells harboring such events could be eliminated by apoptosis or other cell death mechanisms. Lastly, these studies were performed in wild-type mice. Perhaps cumulative damage in neurons is contingent on other susceptibility factors, such as exposure to environmental genotoxins or polymorphisms in genes involved in either the metabolism of these toxins 
or other cellular functions. In any case, whether oxidative stress induces mutations that would result in cell cycle reentry of aging neurons is unclear.

Another potential indirect mechanism for oxidative stress in the induction of aberrant cell cycle reentry is through histone deacetylation. Under normal circumstances, an increase in histone deacetylase activity has been associated with transcriptional repression (63). In agreement, treatment of granule cell cultures with histone deacetylase inhibitors led to an increase in E2F-1 transcription (64). This increase was associated with a rise in the levels of cyclin $\mathrm{E}$ and the cell death molecules APAF-1 and caspase-3, recently shown to be a direct transcriptional target of E2F-1 $(64,65)$. Oxidative stress has been shown to decrease the activity of histone deacetylases 1-10 (66). This change in deacetylase activity could lead to a global inactivation of transcriptional repressors, leading to activation of numerous genes (including cell cycle-inducing genes) and subsequent cell death. An $\mathrm{NAD}^{+}$-dependent histone deacetylase, SIR2, may make an important contribution to longevity in yeast by regulating metabolism and chromatin silencing (67). The homolog of the Sir2 gene, named $\operatorname{SIR} 2 \alpha$, has been found in numerous mammals, including mice. Because of the dependence of SIR $2 \alpha$ activity on its cofactor $\mathrm{NAD}^{+}$, decreases in $\mathrm{NAD}^{+}$levels, occurring because of disruption of metabolic processes, would limit SIR $2 \alpha$ activity. This could be one mechanism by which cell cycle reentry is mediated in the $\mathrm{Hq}$ mutant mouse. Decreased levels of Aif, an NADH oxidase, would decrease the amounts of $\mathrm{NAD}^{+}$available for metabolic processes. In addition, mitochondrial damage would further decrease the available amounts of $\mathrm{NAD}^{+}$. This in turn, could decrease the activity of SIR $2 \alpha$ and result in an increase of transcriptional activation, thereby leading to aberrant cell cycle reentry. Furthermore, SIR2 has been observed to negatively regulate p53 to promote cell survival under conditions of stress - including oxidative stress (68). Therefore, decreased SIR $2 \alpha$ activity could result in an upregulation of p53 activity, which can in turn signal to downstream effectors of the cell cycle (see below).

Other evidence suggests that oxidative stress may more directly trigger unscheduled cell cycle reentry. While exposure of cells to moderate concentrations of hydrogen peroxide induces growth arrest, and exposure to high concentrations induces apoptosis and/or necrosis, low amounts of $\mathrm{H}_{2} \mathrm{O}_{2}$ have been shown to stimulate cell proliferation (69). One potential mechanism by which oxidative stress could induce cell proliferation is through oxidative stimulation of mitogenic pathways. Indeed, ROS have been implicated in cell signaling, specifically through mitogens $(6,70,71)$.

In non-neuronal systems, oxidative stress has been shown to upregulate growth factors including EGF and VEGF (72). Oxidative stress stimulation of growth factor signaling could also be ligand independent. For example, $\mathrm{H}_{2} \mathrm{O}_{2}$ treatment of cell lines or cultured $\mathrm{T}$ cells has been shown to quickly induce tyrosine phosphorylation of multiple proteins including EGFR, PDGFR, and the $T$ cell receptor complex $(73,74)$. The transactivation of these receptors occurs in the

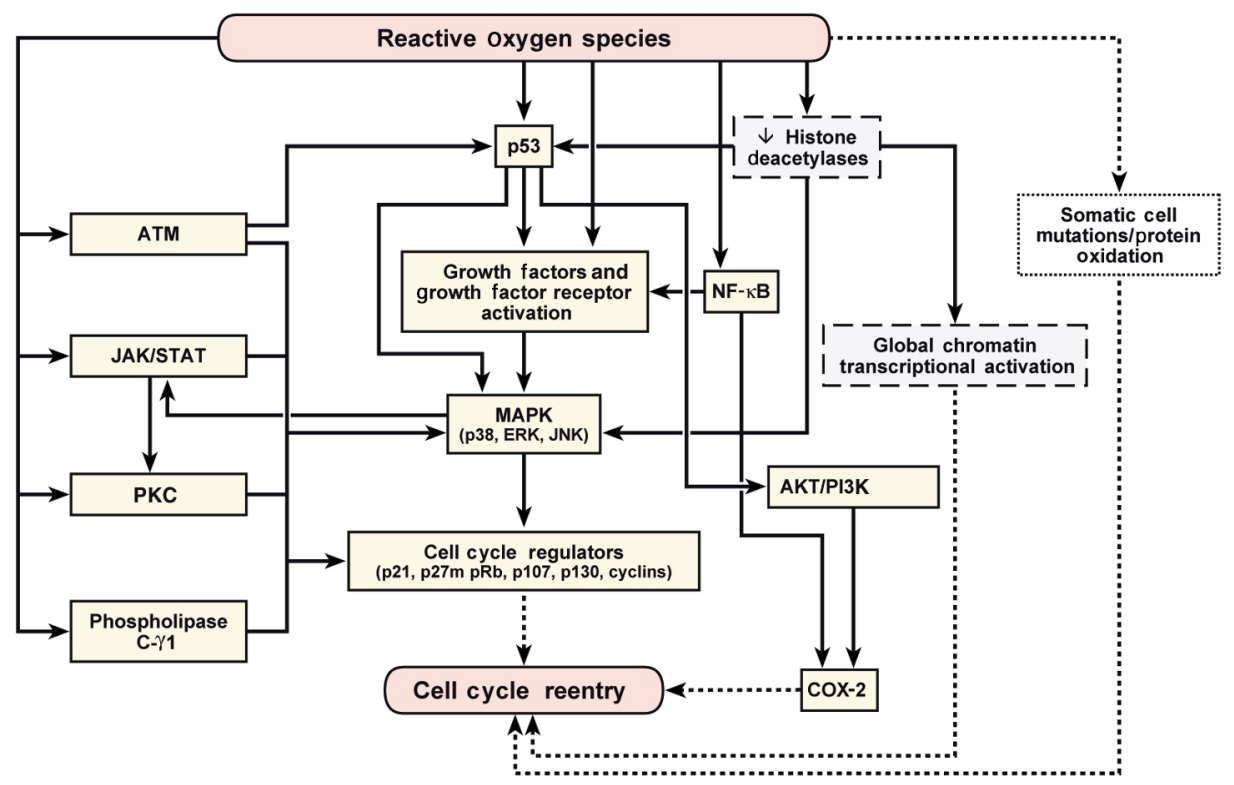

Figure 2

Possible mechanisms for the induction of unscheduled cell cycle reentry in terminally differentiated neurons by oxidative stress. We suggest that oxidative stress, mediated through ROS, could lead to aberrant cell cycle reentry through several different pathways. First, the presence of ROS can result in either cumulative DNA or protein damage that could result in cell cycle reentry. Second, a decrease in histone deacetylase activity can result in chromatin remodeling, leading to transcriptional activation of genes, the products of some of which may lead to cell cycle reentry. Third, many different ROS-activated pathways that have been previously shown to influence the cell cycle in non-neuronal cells could directly or indirectly manipulate a quiescent G0 neuron back into the cell cycle. Several of these pathways also appear to be activated in neurodegenerative disorders. Considerable cross-talk exists between these pathways, as indicated by arrows. Solid arrows represent published pathways, while broken arrows represent hypothesized mechanisms of action. 
absence of ligand and, in the case of EGF receptor, does not result in the endocytosis of the receptor; thus receptor signaling is prolonged (75). Such enhanced signaling has been demonstrated to augment cellular proliferation and transformation.

The transactivation of growth factor receptors appears to signal through several pathways, including the extracellular signal-regulated kinases (ERKs; a subset of the MAPKs), PI3K/AKT, and phospholipase C- $\gamma 1$ (74). The activation of each of these signaling pathways has been shown to induce either apoptosis or cell survival depending on the cell type and oxidative insult. Several other enzymatic pathways are modulated either directly or indirectly by oxidative stress. These include the stress-activated protein kinases JNK and p38, JAK/STAT, PKC, and ataxia telangiectasia mutated, which also participate in signals leading to either cell survival or cell death (74). There is significant cross-talk between these signals, which complicates the task of establishing pathways of oxidative stress-induced apoptosis, and presumably also oxidative stress-induced cell cycle reentry (74) (Figure 2).

ROS can also activate the transcription factor and tumor suppressor protein p53 (76). Although this protein plays an important role as a sensor of genotoxic stress and regulator of genes necessary for growth arrest and cell death, recent evidence suggests that p53 activation can in turn activate genes, including antioxidants and heparin-binding EGF-like factor, that function in compensatory survival pathways (77). p53 can also activate ERK and AKT, leading to an induction of COX-2, an important mediator of various proliferative diseases, including cancer (77).

In neuronal cultures, some evidence supports the rapid upregulation of cell cycle-related genes by oxidative stress. Dopamine, the oxidative metabolism of which generates free radicals, or hydrogen peroxide treatment of postmitotic chick sympathetic neurons, resulted in the expression of cell cycle-related genes, including cyclin B and CDK5, prior to apoptosis (78). Treatment of these cells with antioxidants disrupted both the rise in cell cycle proteins and the subsequent apoptosis. Low potassium levels in cerebellar granule cell cultures induce ROS generation and apoptosis, concomitant with the activation of MAPKK7, one of the activators of the JNK pathway (79). In addition, upregulation of MAPKs, including ERK1/2 and JNK, as well as the serine-threonine kinase Akt, has been observed in $\mathrm{H}_{2} \mathrm{O}_{2}$-treated cultured cortical neurons (80).

ROS and/or oxidative damage can activate gene transcription. However, as discussed previously, transcribed genes may be implicated in either cell survival or cell death. For example, increased NF-кB activity has been observed in $\mathrm{AD}$ patient brains, in both neurons and astrocytes. Although the direct activation of NF- $\mathrm{\kappa B}$ by ROS is controversial (81), the activities of this transcription factor can lend itself to pro- and antiapoptotic roles in the cell. In neurons, the activation of NF- $\mathrm{KB}$ has been linked to the activation of growth factors and COX-2 as well as antioxidants, specifically SOD2. In contrast, NF- $\kappa \mathrm{B}$ activation in astrocytes and microglia results in the activation of pro-oxidants, including nitric oxide, in addition to growth factors (82). Therefore, the activation of NF-KB by ROS in the brain could lead either to cell survival or to signaling that could result in aberrant cell cycle reentry.

Expression analysis of postmortem brains from $\mathrm{AD}$ patients has demonstrated activation of several of these ROS-activated signaling pathways. EGFRimmunoreactive neuritic plaques in the cerebral cortex and hippocampus have been observed (83). This staining was observed mainly in neurons in the periphery of the plaques and correlated with the deposition of paired helical filaments. Expression of the small GTPases RAC and CDC42 has been also observed in neurons in $\mathrm{AD}$ patients (84). These molecules are components of the main signaling pathway between external stressors (such as oxidative stress) and the MAPK pathways. In addition, both RAC and CDC42 have been implicated in progression of the cell cycle, providing a potential link between oxidative stress and cell cycle reentry (82). MAPK p38 expression has been localized to tau-positive neurofibrillary tangles (85, 86). Since p38 has previously been implicated in cell proliferation, apoptosis, and differentiation, this finding could also provide a link between oxidative stress and cell cycle reentry in AD brains.

Lastly, oxidative stress is well known for causing protein damage via nitration or oxidation (23). Such protein modifications have been repeatedly shown in postmortem brain tissue from patients with neurodegenerative disorders, and these reactions can occur generally or have protein specificity. Oxidation could gradually disable proteins necessary for cell cycle repression.

In summary, although unscheduled cell cycle reentry may be mediated by many different insults, evidence is mounting that both cell cycle and oxidative stress may be team players in neurodegenerative disorders. We have discussed some of the potential pathways by which the repression of the cell cycle in neurons may be modulated by ROS (Figure 2). Given the distinctive pathology of different neurodegenerative diseases, it is likely that specific types of neurons may respond to different signaling pathways, or need activation of multiple signaling pathways. The testing of the roles of these and other potential pathways in neurodegeneration will require the development and analysis of additional animal models, in addition to further correlative human studies. However, the careful dissection of the interplay between oxidative stress and the cell cycle will greatly advance our understanding of several debilitating neurodegenerative disorders.

\section{Acknowledgments}

We thank Edward Leiter for comments on the manuscript and Jennifer Smith for graphical assistance with the manuscript figures. This work was supported by NIH grants AG-19358 and NS-42613 to S.L. Ackerman.

\footnotetext{
1. Nagy, Z., Esiri, M.M., and Smith, A.D. 1998. The cell division cycle and the pathophysiology of Alzheimer's disease. Neuroscience. 87:731-739. 2. Nagy, Z. 1999. Mechanisms of neuronal death in Down's syndrome. J. Neural Transm. Suppl. 57:233-245.

3. Herrup, K., and Busser, J.C. 1995. The induction of multiple cell cycle events precedes target-related neuronal death. Development. 121:2385-2395.

4. Migheli, A., et al. 1999. A cell cycle alteration precedes apoptosis of
} 
granule cell precursors in the weaver mouse cerebellum. Am. J. Pathol. 155:365-373.

5. Klein, J.A., et al. 2002. The harlequin mouse mutation downregulates apoptosis-inducing factor. Nature. 419:367-374.

6. Kannan, K., and Jain, S.K. 2000. Oxidative stress and apoptosis. Pathophysiology. 7:153-163.

7. Leonardi, E.T., and Mytilineou, C. 1998. Cell culture models of neuronal degeneration and neuroprotection. Implications for Parkinson's disease. Adv. Exp. Med. Biol. 446:203-222.

8. Yokota, T., et al. 2001. Delayed-onset ataxia in mice lacking alpha-tocopherol transfer protein: model for neuronal degeneration caused by chronic oxidative stress. Proc. Natl. Acad. Sci. U. S. A. 98:15185-15190.

9. Melov, S., et al. 1998. A novel neurological phenotype in mice lacking mitochondrial manganese superoxide dismutase. Nat. Genet. 18:159-163.

10. Sayre, L.M., Smith, M.A., and Perry, G. 2001. Chemistry and biochemistry of oxidative stress in neurodegenerative disease. Curr. Med. Chem. 8:721-738.

11. Aksenov, M.Y., et al. 1998. The expression of key oxidative stress-handling genes in different brain regions in Alzheimer's disease. J. Mol. Neurosci. 11:151-164.

12. Hensley, K., et al. 1994. A model for beta-amyloid aggregation and neurotoxicity based on free radical generation by the peptide: relevance to Alzheimer disease. Proc. Natl. Acad. Sci. U. S. A. 91:3270-3274.

13. Jang, J.H., and Surh, Y.J. 2002. Beta-amyloid induces oxidative DNA damage and cell death through activation of c-Jun $\mathrm{N}$ terminal kinase. Ann. N. Y. Acad. Sci. 973:228-236.

14. Matsuoka, Y., Picciano, M., La Francois, J., and Duff, K. 2001. Fibrillar beta-amyloid evokes oxidative damage in a transgenic mouse model of Alzheimer's disease. Neuroscience. 104:609-613

15. Smith, M.A., Rottkamp, C.A., Nunomura, A., Raina, A.K., and Perry, G. 2000. Oxidative stress in Alzheimer's disease. Biochim. Biophys. Acta. 1502:139-144

16. Jenner, P., and Olanow, C.W. 1996. Oxidative stress and the pathogenesis of Parkinson's disease. Neurology. 47(Suppl. 3):S161-S170.

17. Giasson, B.I., Ischiropoulos, H., Lee, V.M., and Trojanowski, J.Q. 2002. The relationship between oxidative/nitrative stress and pathological inclusions in Alzheimer's and Parkinson's diseases. Free Radic. Biol. Med. 32:1264-1275.

18. Fukushima, T., et al. 1995. Radical formation site of cerebral complex I and Parkinson's disease. J. Neurosci. Res. 42:385-390.

19. Cassarino, D.S., et al. 1997. Elevated reactive oxygen species and antioxidant enzyme activities in animal and cellular models of Parkinson's disease. Biochim. Biophys. Acta. 1362:77-86.

20. Sherer, T.B., et al. 2002. An in vitro model of Parkinson's disease: linking mitochondrial impairment to altered alpha-synuclein metabolism and oxidative damage. J. Neurosci. 22:7006-7015.

21. Chun, H.S., et al. 2001. Dopaminergic cell death induced by MPP $(+)$, oxidant and specific neurotoxicants shares the common molecular mechanism. J. Neurochem. 76:1010-1021.

22. Albers, D.S., and Augood, S.J. 2001. New insights into progressive supranuclear palsy. Trends Neurosci. 24:347-353.

23. Butterfield, D.A., and Kanski, J. 2001. Brain protein oxidation in agerelated neurodegenerative disorders that are associated with aggregated proteins. Mech. Ageing Dev. 122:945-962.

24. Kim, J.I., et al. 2001. Oxidative stress and neurodegeneration in prion diseases. Ann. N. Y. Acad. Sci. 928:182-186.

25. Cassarino, D.S., and Bennett, J.P., Jr. 1999. An evaluation of the role of mitochondria in neurodegenerative diseases: mitochondrial mutations and oxidative pathology, protective nuclear responses, and cell death in neurodegeneration. Brain Res. Brain Res. Rev. 29:1-25.

26. Heintz, N. 1993. Cell death and the cell cycle: a relationship between transformation and neurodegeneration? Trends Biochem. Sci. 18:157-159.

27. Feddersen, R.M., Ehlenfeldt, R., Yunis, W.S., Clark, H.B., and Orr, H.T. 1992. Disrupted cerebellar cortical development and progressive degeneration of Purkinje cells in SV40 T antigen transgenic mice. Neuron. 9:955-966

28. Frade, J.M. 2000. Unscheduled re-entry into the cell cycle induced by NGF precedes cell death in nascent retinal neurones. J. Cell Sci. 113:1139-1148.

29. Verdaguer, E., et al. 2002. Kainic acid-induced apoptosis in cerebellar granule neurons: an attempt at cell cycle re-entry. Neuroreport. 13:413-416.

30. Copani, A., et al. 1999. Mitotic signaling by beta-amyloid causes neuronal death. FASEB J. 13:2225-2234.

31. Copani, A., et al. 2002. Beta-amyloid-induced synthesis of the ganglioside GD3 is a requisite for cell cycle reactivation and apoptosis in neurons. J. Neurosci. 22:3963-3968.

32. Feddersen, R.M., Clark, H.B., Yunis, W.S., and Orr, H.T. 1995. In vivo viability of postmitotic Purkinje neurons requires pRb family member function. Mol. Cell. Neurosci. 6:153-167.

33. Al-Ubaidi, M.R., et al. 1997. Unscheduled DNA replication precedes apoptosis of photoreceptors expressing SV40 T antigen. Exp. Eye Res. 64:573-585
34. ElShamy, W.M., Fridvall, L.K., and Ernfors, P. 1998. Growth arrest failure, G1 restriction point override, and S phase death of sensory precursor cells in the absence of neurotrophin-3. Neuron. 21:1003-1015.

35. Katchanov, J., et al. 2001. Mild cerebral ischemia induces loss of cyclindependent kinase inhibitors and activation of cell cycle machinery before delayed neuronal cell death. J. Neurosci. 21:5045-5053.

36. Osuga, H., et al. 2000. Cyclin-dependent kinases as a therapeutic target for stroke. Proc. Natl. Acad. Sci. U. S. A. 97:10254-10259.

37. Rangathan, S., and Bowser, R. 2003. Alterations in G(1) to S phase cellcycle regulators during amyotrophic lateral sclerosis. Am. J. Pathol. 162:823-835.

38. McShea, A., Harris, P.L., Webster, K.R., Wahl, A.F., and Smith, M.A. 1997 Abnormal expression of the cell cycle regulators P16 and CDK4 in Alzheimer's disease. Am. J. Pathol. 150:1933-1939.

39. Busser, J., Geldmacher, D.S., and Herrup, K. 1998. Ectopic cell cycle proteins predict the sites of neuronal cell death in Alzheimer's disease brain. J. Neurosci. 18:2801-2807.

40. Herrup, K., and Arendt, T. 2002. Re-expression of cell cycle proteins induces neuronal cell death during Alzheimer's disease. J. Alzheimers Dis. 4:243-247.

41. Yang, Y., Geldmacher, D.S., and Herrup, K. 2001. DNA replication precedes neuronal cell death in Alzheimer's disease. J. Neurosci. 21:2661-2668.

42. Nagy, Z., Esiri, M.M., and Smith, A.D. 1997. Expression of cell division markers in the hippocampus in Alzheimer's disease and other neurodegenerative conditions. Acta Neuropathol. (Berl.) 93:294-300.

43. Konishi, Y., Lehtinen, M., Donovan, N., and Bonni, A. 2002. Cdc2 phosphorylation of BAD links the cell cycle to the cell death machinery. Mol. Cell. 9:1005-1016.

44. Migliore, L., and Coppede, F. 2002. Genetic and environmental factors in cancer and neurodegenerative diseases. Mutat. Res. 512:135-153.

45. Chen, Q.M., Liu, J., and Merrett, J.B. 2000. Apoptosis or senescence-like growth arrest: influence of cell-cycle position, p53, p21 and bax in $\mathrm{H} 2 \mathrm{O} 2$ response of normal human fibroblasts. Biochem. J. 347:543-551.

46. Barber, B.R. 1971. Research news. Mouse News Letter. 45:34-35.

47. Bronson, R.T., Lane, P.W., Harris, B.S., and Davisson, M.T. 1990. Harlequin $(\mathrm{Hq})$ produces progressive cerebellar atrophy. Mouse Genome. 87:110.

48. Susin, S.A., et al. 1999. Molecular characterization of mitochondrial apoptosis-inducing factor. Nature. 397:441-446.

49. Mate, M.J., et al. 2002. The crystal structure of the mouse apoptosisinducing factor AIF. Nat. Struct. Biol. 9:442-446.

50. Cande, C., Cecconi, F., Dessen, P., and Kroemer, G. 2002. Apoptosisinducing factor (AIF): key to the conserved caspase-independent pathways of cell death? J. Cell Sci. 115:4727-4734.

51. Miramar, M.D., et al. 2001. NADH oxidase activity of mitochondrial apoptosis-inducing factor. J Biol. Chem. 276:16391-16398.

52. Wang, X., Yang, C., Chai, J., Shi, Y., and Xue, D. 2002. Mechanisms of AIF-mediated apoptotic DNA degradation in Caenorhabditis elegans. Science. 298:1587-1592.

53. Arnoult, D., et al. 2002. Mitochondrial release of apoptosis-inducing factor occurs downstream of cytochrome $\mathrm{c}$ release in response to several proapoptotic stimuli. J. Cell Biol. 159:923-929.

54. Joza, N., et al. 2001. Essential role of the mitochondrial apoptosis-inducing factor in programmed cell death. Nature. 410:549-554.

55. Bonni, A. 2003. Neurodegeneration: a non-apoptotic role for AIF in the brain. Curr. Biol. 13:R19-R21.

56. Lipton, S.A., and Bossy-Wetzel, E. 2002. Dueling activities of AIF in cell death versus survival: DNA binding and redox activity. Cell. 111:147-150.

57. Holmgren, A. 2000. Antioxidant function of thioredoxin and glutaredoxin systems. Antioxid. Redox Signal. 2:811-820.

58. White, A.R., et al. 1998. Survival of cultured neurons from amyloid precursor protein knock-out mice against Alzheimer's amyloid-beta toxicity and oxidative stress. J. Neurosci. 18:6207-6217.

59. Turker, M.S. 2000. Somatic cell mutations: can they provide a link between aging and cancer? Mech. Ageing Dev. 117:1-19.

60. Halliwell, B. 1998. Can oxidative DNA damage be used as a biomarker of cancer risk in humans? Problems, resolutions and preliminary results from nutritional supplementation studies. Free Radic. Res. 29:469-486.

61. Sekiguchi, M., and Tsuzuki, T. 2002. Oxidative nucleotide damage: consequences and prevention. Oncogene. 21:8895-8904.

62. Dolle, M.E., et al. 1997. Rapid accumulation of genome rearrangements in liver but not in brain of old mice. Nat. Genet. 17:431-434.

63. Pazin, M.J., and Kadonaga, J.T. 1997. What's up and down with histone deacetylation and transcription? Cell. 89:325-328.

64. Boutillier, A.L., Trinh, E., and Loeffler, J.P. 2003. Selective E2F-dependent gene transcription is controlled by histone deacetylase activity during neuronal apoptosis. J. Neurochem. 84:814-828.

65. Nahle, Z., et al. 2002. Direct coupling of the cell cycle and cell death machinery by E2F. Nat. Cell Biol. 4:859-864.

66. Rahman, I. 2002. Oxidative stress, transcription factors and chromatin remodeling in lung inflammation. Biochem. Pharmacol. 64:935-942.

67. Guarente, L. 2000. Sir2 links chromatin silencing, metabolism, and aging. Genes Dev. 14:1021-1026. 
68. Luo, J., et al. 2001. Negative control of p53 by Sir2alpha promotes cell survival under stress. Cell. 107:137-148.

69. Clement, M.V., and Pervaiz, S. 1999. Reactive oxygen intermediates regulate cellular response to apoptotic stimuli: an hypothesis. Free Radic. Res. 30:247-252.

70. Chan, P.H. 2001. Reactive oxygen radicals in signaling and damage in the ischemic brain. J. Cereb. Blood Flow Metab. 21:2-14.

71. Droge, W. 2002. Free radicals in the physiological control of cell function. Physiol. Rev. 82:47-95.

72. Maulik, N., and Das, D.K. 2002. Redox signaling in vascular angiogenesis. Free Radic. Biol. Med. 33:1047-1060.

73. Huang, R.P., Wu, J.X., Fan, Y., and Adamson, E.D. 1996. UV activates growth factor receptors via reactive oxygen intermediates. J. Cell Biol. 133:211-220.

74. Martindale, J.L., and Holbrook, N.J. 2002. Cellular response to oxidative stress: signaling for suicide and survival. J. Cell. Physiol. 192:1-15.

75. Ravid, T., Sweeney, C., Gee, P., Carraway, K.L., 3rd, and Goldkorn, T. 2002. Epidermal growth factor receptor activation under oxidative stress fails to promote c-Cbl mediated down-regulation. J. Biol. Chem. 277:31214-31219.

76. Nakamura, T., and Sakamoto, K. 2001. Reactive oxygen species up-regulates cyclooxygenase-2, p53, and Bax mRNA expression in bovine luteal cells. Biochem. Biophys. Res. Commun. 284:203-210.

77. Han, J.A., et al. 2002. P53-mediated induction of Cox- 2 counteracts p53or genotoxic stress-induced apoptosis. EMBO J. 21:5635-5644.
78. Shirvan, A., et al. 1998. Expression of cell cycle-related genes during neuronal apoptosis: is there a distinct pattern? Neurochem. Res. 23:767-777.

79. Trotter, L., et al. 2002. Mitogen-activated protein kinase kinase 7 is activated during low potassium-induced apoptosis in rat cerebellar granule neurons. Neurosci. Lett. 320:29-32.

80. Crossthwaite, A.J., Hasan, S., and Williams, R.J. 2002. Hydrogen peroxide-mediated phosphorylation of ERK1/2, Akt/PKB and JNK in cortical neurones: dependence on $\mathrm{Ca}(2+)$ and PI3-kinase. J. Neurochem. 80:24-35.

81. Li, N., and Karin, M. 1999. Is NF-kappaB the sensor of oxidative stress? FASEB J. 13:1137-1143.

82. Mattson, M.P., and Camandola, S. 2001. NF-кB in neuronal plasticity and neurodegenerative disorders. J. Clin. Invest. 107:247-254.

83. Birecree, E., Whetsell, W.O., Jr., Stoscheck, C., King, L.E., Jr., and Nanney, L.B. 1988. Immunoreactive epidermal growth factor receptors in neuritic plaques from patients with Alzheimer's disease. J. Neuropathol. Exp. Neurol. 47:549-560.

84. Zhu, X., et al. 2000. Activation of oncogenic pathways in degenerating neurons in Alzheimer disease. Int. J. Dev. Neurosci. 18:433-437.

85. Zhu, X., et al. 2000. Activation of p38 kinase links tau phosphorylation, oxidative stress, and cell cycle-related events in Alzheimer disease. J. Neuropathol. Exp. Neurol. 59:880-888.

86. Atzori, C., et al. 2001. Activation of the JNK/p38 pathway occurs in diseases characterized by tau protein pathology and is related to tau phosphorylation but not to apoptosis. J. Neuropathol. Exp. Neurol. 60:1190-1197. 